\title{
The diocotron instability in a pulsar cylindrical electrosphere
}

\author{
J. Pétri
}

\author{
Max-Planck-Institut für Kernphysik, Saupfercheckweg 1, 69117 Heidelberg, Germany \\ e-mail: jerome.Petri@mpi-hd.mpg.de
}

Received 25 August 2006 / Accepted 1 November 2006

\begin{abstract}
Context. The physics of the pulsar inner magnetosphere remains poorly constrained by observations. Although about 2000 pulsars have been discovered to date, little is known about their emission mechanism. Large vacuum gaps probably exist and a non-neutral plasma made of electrons in some regions and of positrons in some other regions fills space to form an electrosphere.

Aims. The purpose of this work is to study the stability properties of the differentially rotating equatorial disk in the pulsar's electrosphere for which the magnetic field is assumed to be dipolar. In contrast to previous studies, the magnetic field is not restricted to being uniform.

Methods. A pseudo-spectral Galerkin method using Tchebyshev-polynomial expansion is developed to compute the spectrum of the diocotron instability in a non-neutral plasma column confined between two cylindrically conducting walls. Moreover, the inner wall carries a given charge per unit length in order to account for the presence of a charged neutron star at the centre of the electrosphere. Results. We show several eigenfunctions and eigenspectra obtained for different initial density profiles and electromagnetic field configurations useful for laboratory plasmas. The algorithm is very efficient in computing the fastest growing modes. Applications to a "cylindrical" electrosphere are also shown for several differential rotation profiles. It is found that the growth rates of the diocotron instability have the same order of magnitude as the rotation rate.

Conclusions. The instability develops on a very short timescale and can account for very efficient particle diffusion across the magnetic field lines, as already claimed in a previous work. The exact geometry of the confined plasma, whether a thin disk or a cylinder, does not significantly affect the spectrum of the diocotron instability.
\end{abstract}

Key words. instabilities - methods: analytical - methods: numerical - stars: neutron

\section{Introduction}

The detailed structure of charge distribution and electric-current circulation in the closed magnetosphere of a pulsar remains poorly understood. Although it is often assumed that the plasma fills the space entirely and corotates with the neutron star, it is on the contrary very likely that it only partly fills it, leaving large vacuum gaps between plasma-filled regions. The existence of such gaps in aligned rotators has been very clearly established by Krause-Polstorff \& Michel (1985a,b). Since then, a number of different numerical approaches to the problem have confirmed their conclusions, including some work by Rylov (1989), Shibata (1989), Zachariades (1993), Neukirch (1993), Thielheim \& Wolfsteller (1994), Spitkovsky \& Arons (2002), and by ourselves (Pétri et al. 2002a). This conclusion about the existence of vacuum gaps has been reached from a self-consistent solution of Maxwell's equations in the case of the aligned rotator. Moreover, Smith et al. (2001) have shown by numerical modeling that an initially filled magnetosphere like the Goldreich-Julian model evolves by opening up large gaps and stabilises to the partially filled and partially void solution found by Krause-Polstorff \& Michel (1985a), and also by Pétri et al. (2002a). The status of models of the pulsar magnetospheres, or electrospheres, has recently been critically reviewed by Michel (2005). A solution with vacuum gaps has the peculiar property that those parts of the magnetosphere that are separated (following magnetic field lines) from the star's surface by a vacuum region are not corotating and so suffer differential rotation.
This raises the question of the stability of such a charged plasma flow. The differential rotation in the equatorial, non neutral disk induces the so-called diocotron and magnetron instabilities that are well known to plasma physicists (O'Neil 1980; Davidson 1990; O'Neil \& Smith 1992). In the inner parts of the magnetosphere, far from the light cylinder, the instability reduces to its electrostatic form, the diocotron instability. The linear development of the diocotron instability of a thin differentially rotating charged disk was studied by Pétri et al. (2002b) and shown to proceed at a growth rate comparable to the star's rotation rate. The non linear development of this instability was studied by Pétri et al. (2003), still in the framework of an infinitely thin disk model. When there is no external source of charges feeding the magnetosphere, it was found that the plasma remains confined in regions close to the equilibrium state, as also shown by Aly (2005). When however the disk can be fed by some charge source, Pétri et al. (2003) have shown that the instability causes a cross-field transport of these charges in the equatorial disk, evolving into a net out-flowing flux of charges. Spitkovsky \& Arons (2002) have numerically studied the problem, and concluded that this charge transport tends to fill the gaps with plasma. Since a filled magnetosphere is stable to the diocotron instability, it seems unlikely that the instability could indeed bring the system to a completely filled state, even though the disk would not remain thin, as assumed by Pétri et al. (2002b, 2003). It should be noted that the appearance of a cross-field electric current as a result of the diocotron instability has been observed by Pasquini \& Fajans (2002) in laboratory experiments 
in which charged particles were continuously injected in the plasma column trapped in a Malmberg-Penning configuration.

The aim of this work is to show that the differentially rotating charged disk is not infinitely thin does not invalidate our former conclusion (Pétri et al. 2002b) that the growth rate of the instability is as fast as the star's rotation rate. The antithetical situation for a thin disk is that of an infinitely thick disk. By this we mean a plasma column, the structure of which does not vary with the cylindrical coordinate $z$ and in which the magnetic field depends on the cylindrical coordinate $r$ and is oriented parallel to the $z$ direction. Whereas the thin disk model can conveniently describe perturbations, the horizontal size of which is much larger than the disk's thickness, the (infinitely) thick model would be more appropriate to perturbations whose the horizontal size is much less than the disk's thickness. In the electrostatic approximation, the magnetic field is not altered by the perturbations so it remains straight. From the inertia-less approximation, the charged fluid velocity is given by Eq. (17), which implies that $\boldsymbol{E}+\boldsymbol{v} \times \boldsymbol{B}=0$ and then that $\boldsymbol{E} \cdot \boldsymbol{B}=0$. As a result, the perturbed electric field and flow are 2-dimensional, since the electric potential perturbation is independent of $z$. It should be kept in mind that the infinitely-thick disk model with a $z$-aligned magnetic field is in fact meant to represent a disk of a finite thickness embedded in a 2D potential magnetic field. Such 2D fields, like

$\boldsymbol{B}=B_{r}(r, z) \boldsymbol{e}_{r}+B_{z}(r, z) \boldsymbol{e}_{z}$

as opposed to 1D fields like $\boldsymbol{B}=B_{z}(r) \boldsymbol{e}_{z}$, may be current-free and still have a radial dependence on $B_{z}(r, 0)$ in the equatorial plane. In the 1D model, the disk, though not infinitely thin, is regarded as not being thick enough for the radial field component $B_{r}(r, z)$ to play a role. The component $B_{z}(r, 0)$ is taken as an approximation to $B_{z}(r, z)$. The domain of validity of a 1D model then is that $B_{r}(r, z)$ remains small in the disk, while the horizontal size of the perturbations is less than the disk's thickness. The real situation is between the infinitely thin and the infinitely thick disk model. Dealing with a finite thickness disk would involve considering the 2D magnetic field structure and numerically calculating the solution of the Poisson equation in the appropriate geometry. Obtaining this solution was the most time-consuming part of the calculations in Pétri et al. (2003) and things would be even worse if a 2D geometry with a thick disk were to be considered. Adopting a 1D unperturbed geometry model considerably alleviates such difficulties and seems appropriate for reaching a conclusion about the range in which the growth rates of the diocotron instability are to be found when the infinitely thin disk approximation is relaxed.

In this paper we present a numerical analysis of the linear growth of the diocotron instability in the 1D model. We plan to compute the full non linear development of the instability in the same geometry, by using a 2D electrostatic PIC code, a study that would also be relevant to laboratory setups.

The diocotron spectrum for cylindrical geometry and uniform external magnetic field has been investigated analytically by Levy (1965) and numerically by Goswami et al. (1999), who used a finite difference scheme. Variational techniques for toroidal non-neutral plasmas have also been used; see Bhattacharyya (2000).

The paper is organised as follows. In Sect. 2, we describe the initial setup of the plasma column in the laboratory consisting of an axially symmetric equilibrium between two conducting walls. The slightly different approach used for the pulsar's electrosphere is also described. In Sect. 3, we recall the generalised linear eigenvalue problem satisfied by the perturbed electric potential. Next, in Sect. 4, the pseudo-spectral Galerkin numerical algorithm for computing the diocotron spectrum is presented. Finally, some typical spectra for laboratory plasma are shown in Sect. 5. We discuss the application to electrospheric plasmas in more detail, which is the main aim of this work. The conclusions and the possible generalisation are presented in Sect. 6 .

\section{Initial setup}

In laboratory plasmas, the motion of particles is imposed by external devices controlling the potential and electric field. It is the most common situation. However, since we intend to apply the algorithm to the pulsar's electrosphere, it is also interesting to study the stability properties of non-neutral plasmas by imposing the rotation profile instead of the density profile. Therefore, we first describe the case encountered in the laboratory and then discussed how to apply and extend it to real pulsars.

\subsection{Laboratory plasma}

We consider a single-species non-neutral plasma consisting of particles with mass $m_{\mathrm{e}}$ and charge $q$ trapped between two cylindrically conducting walls located at $r=W_{1}$ and $r=W_{2}>W_{1}$. The plasma column itself is confined between $R_{1} \geq W_{1}$ and $R_{2} \leq W_{2}$. This allows us to take vacuum regions into account between the plasma and the conducting walls. We adopt cylindrical coordinates denoted by $(r, \varphi, z)$ and the corresponding basis vectors $\left(\boldsymbol{e}_{r}, \boldsymbol{e}_{\varphi}, \boldsymbol{e}_{z}\right)$. In order to simulate the presence of a charged neutron star generating a radial electric field, the inner wall at $W_{1}$ carries a charge $Q$ per unit length such that its electric field is simply given by the Maxwell-Gauss theorems

$\boldsymbol{E}_{\mathrm{w}}(r)=\frac{Q}{2 \pi \varepsilon_{0} r} \boldsymbol{e}_{r}$

In the equilibrium configuration, the particle number density is $n(r)$ and the charge density is $\rho(r)=q n(r)$. In contrast to earlier studies, the external magnetic field, along the $z$-axis, is not necessarily uniform:

$\boldsymbol{B}=B_{z}(r) \boldsymbol{e}_{z}$

Strictly speaking, this magnetic field is associated with an azimuthal current because $\boldsymbol{\nabla} \wedge \boldsymbol{B} \neq \boldsymbol{0}$. However, as claimed in the introduction, there is also a (weak) radial component, Eq. (1), which should compensate for this current. Let us find the conditions for which approximation Eq. (3) holds, assuming a power law for $B_{z}$, Eq. (48). From Eq. (1), the $\varphi$-component of the curl reads

$\frac{\partial B_{r}}{\partial z}-\frac{\partial B_{z}}{\partial r}=\frac{\partial B_{r}}{\partial z}+\alpha \frac{B_{z}}{r}$

With the boundary conditions $B_{r}(r, z=0)=0$, we find that near the equatorial plane

$B_{r}=-\alpha \frac{z}{r} B_{z}$

where $\alpha$ is of order unity, the radial component of the magnetic field is negligible whenever $z \ll r$. Therefore, our approximation Eq. (3) holds in the thin disk limit (intermediate between infinitely thin and infinitely thick).

The electric field is made of two parts, the first one arising from the plasma column $\boldsymbol{E}_{\mathrm{p}}$ itself, and the second one from the inner conducting wall $\boldsymbol{E}_{\mathrm{w}}$, Eq. (2). We assume that the electric 
field induced by the plasma vanishes at $r=W_{1}$, i.e. $\boldsymbol{E}_{\mathrm{p}}\left(W_{1}\right)=\mathbf{0}$. We therefore get

$\boldsymbol{E}_{\mathrm{p}}(r)=\frac{1}{\varepsilon_{0} r} \int_{W_{1}}^{r} \rho\left(r^{\prime}\right) r^{\prime} \mathrm{d} r^{\prime} \boldsymbol{e}_{r}$.

The total electric field, directed along the radial direction $\boldsymbol{e}_{r}$, is therefore

$\boldsymbol{E}=\boldsymbol{E}_{\mathrm{p}}+\boldsymbol{E}_{\mathrm{w}}=E_{r} \boldsymbol{e}_{r}$

At equilibrium, the plasma is rotating between the two walls at a speed $\Omega(r)$. In the stationary state, the centrifugal force is balanced by the Lorentz force such that

$q\left(E_{r}+r \Omega B_{z}\right)+m_{\mathrm{e}} r \Omega^{2}=0$.

Introducing the cyclotron frequency by $\omega_{\mathrm{c}}=\frac{q B_{z}}{m_{\mathrm{e}}}$ and the plasma frequency by $\omega_{\mathrm{p}}^{2}=\frac{n q^{2}}{m_{\mathrm{e}} \varepsilon_{0}}$, the angular speed of the column satisfies the quadratic equation

$\Omega^{2}+\omega_{\mathrm{c}} \Omega+\frac{q E_{r}}{m_{\mathrm{e}} r}=0$,

leading to two possible solutions for $\Omega$, namely

$\Omega=-\frac{\omega_{\mathrm{c}}}{2}\left[1 \pm \sqrt{1-4 \frac{q E_{r}}{m_{\mathrm{e}} r \omega_{\mathrm{c}}^{2}}}\right]$.

If the electric field is sufficiently weak, i.e. $q E_{r} \ll m_{\mathrm{e}} r \omega_{\mathrm{c}}^{2}$, the solutions are approximated by $\Omega=-\omega_{\mathrm{c}}$ and

$\Omega=-\frac{E_{r}}{r B_{z}}$.

The weak field limit is equivalent to a low-density plasma column because it implies $\omega_{\mathrm{p}}^{2} \ll \omega_{\mathrm{c}}^{2}$. The solution Eq. (11) corresponds to the usual electric drift approximation (Davidson \& Tsang 1984). In the rest of this paper, we retain this assumption; therefore, the equation of motion for the charged particles is replaced by the electric drift approximation. Indeed, we only consider the low-density non-neutral plasma case for which the diocotron regime is valid. Neglecting electron inertia, the electric drift approximation applies. For $Q=0$ and a constant density profile in the plasma column with $W_{1}=0$, it corresponds to a circular motion at the diocotron frequency defined by, see for instance Davidson (1990),

$\omega_{\mathrm{D}}=\frac{\omega_{\mathrm{p}}^{2}}{2 \omega_{\mathrm{c}}}$.

\subsection{Electrospheric plasma}

Our purpose is to study the non-neutral plasma instabilities occurring in the pulsar inner magnetosphere. The most interesting feature obtained in the work by Pétri et al. (2002a) by constructing an electrospheric model was a differential rotation in the positively charged equatorial disk. It can indeed be shown that large vacuum gaps imply a significant departure from corotation. That is why, when applying our algorithm to pulsars, it is more appropriate to assume a given rotation profile in the plasma $\Omega(r)$, instead of densities and potential imposed by external devices. In the electric drift approximation, using the Maxwell-Gauss law, the charge density is recovered according to ideal MHD by

$\boldsymbol{E}=-(\boldsymbol{\Omega} \wedge \boldsymbol{r}) \wedge \boldsymbol{B}$ leading to the charge density as follows

$\rho=-\varepsilon_{0}\left[\frac{B_{z}}{r} \frac{\partial}{\partial r}\left(r^{2} \Omega\right)+r \Omega \frac{\partial B_{z}}{\partial r}\right]$.

Therefore, there are only 2 independent functions, namely the couple $\left(B_{z}, \Omega\right)$ appropriate for pulsar electrospheric plasmas and the couple $\left(B_{z}, \rho\right)$ useful for laboratory plasmas.

\section{Linear analysis}

We consider two different plasma configurations. In the first case, the plasma column is supposed to be in contact with both the inner and the outer walls. This is called a single domain. In the second case, vacuum regions exist between the plasma column and the inner and/or the outer walls. This is called the multi-domain. We introduced these two situations because of the slightly different numerical treatment of the problem. Indeed, vacuum regions are treated analytically, whereas regions filled with plasma are treated numerically.

\subsection{Single domain}

Let us start with a column density in contact with the inner and the outer conducting walls. The motion of the column of plasma is governed by the conservation of charge, the Maxwell-Poisson equation, and the electric drift approximation, respectively:

$\frac{\partial \rho}{\partial t}+\operatorname{div}(\rho \boldsymbol{v})=0$

$\Delta \phi+\frac{\rho}{\varepsilon_{0}}=0$

$\boldsymbol{v}=\frac{\boldsymbol{E} \wedge \boldsymbol{B}}{B^{2}}$

$\boldsymbol{E}=-\boldsymbol{\nabla} \phi$.

We recall that the magnetic field remains constant in time in the diocotron regime. We apply the standard linear perturbation theory. Introducing perturbations of physical quantities $X$ like electric potential, density, and velocity components by the expansion

$X(r, \varphi, t)=X(r) \mathrm{e}^{\mathrm{i}(m \varphi-\omega t)}$,

the eigenvalue problem for the perturbed electric potential $\phi(r)$ is expressed as

$\left[\frac{1}{r} \frac{\partial}{\partial r}\left(r \frac{\partial \phi}{\partial r}\right)-\frac{m^{2}}{r^{2}}\right] \phi=\frac{m}{(\omega-m \Omega)} \frac{1}{\varepsilon_{0} r} \frac{\partial}{\partial r}\left(\frac{\rho}{B_{z}}\right) \phi$

For the purpose of our numerical algorithm, it is more convenient to rewrite it as a generalised linear eigenvalue problem as follows:

$\omega \mathcal{L}_{m}(\phi)=m \Omega \mathcal{L}_{m}(\phi)+q_{m} \phi$.

The Laplacian operator $\mathcal{L}_{m}$ for each azimuthal mode $m$ is given by

$\mathcal{L}_{m}(\phi)=\left[\frac{1}{r} \frac{\partial}{\partial r}\left(r \frac{\partial \phi}{\partial r}\right)-\frac{m^{2}}{r^{2}}\right] \phi$

and the function $q_{m}$ is

$q_{m}=\frac{m}{\varepsilon_{0} r} \frac{\partial}{\partial r}\left(\frac{\rho}{B_{z}}\right)$ 
For the electrospheric plasma, we can eliminate $\rho$ by Eq. (14) and write

$q_{m}=-\frac{m}{r} \frac{\partial}{\partial r}\left[\frac{1}{r} \frac{\partial}{\partial r}\left(r^{2} \Omega\right)+\frac{r \Omega}{B_{\mathrm{z}}} \frac{\partial B_{z}}{\partial r}\right]$.

Note that this function does not depend on the intensity of $B_{z}$ but only on its radial profile. However, it depends linearly on the amplitude of the differential rotation, meaning that strong departure from corotation leads to strong instability, as will be shown in the next section. The eigenvalue problem, Eq. (21), is supplemented by homogeneous boundary conditions such that the perturbed potential vanishes at the boundaries, namely

$\phi\left(W_{1}\right)=\phi\left(W_{2}\right)=0$.

\subsection{Multi-domain decomposition}

When vacuum gaps exist between the walls and the plasma, the discontinuity in the density profile introduces a Gibbs phenomenon and therefore drastically decreases the efficiency of our pseudo-spectral algorithm. To overcome this difficulty, we decompose the space between the two walls into three distinct regions:

- region I: vacuum space between inner wall and inner boundary of the plasma column, with the solution for the electric potential denoted by $\phi_{\mathrm{I}}$, defined for $W_{1} \leq r \leq R_{1}$;

- region II: the plasma column itself located between $R_{1}$ and $R_{2}$, solution denoted by $\phi_{\mathrm{II}}$, defined for $R_{1} \leq r \leq R_{2}$;

- region III: vacuum space between the outer boundary of the plasma column and the outer wall, solution denoted by $\phi_{\mathrm{III}}$, defined for $R_{2} \leq r \leq W_{2}$.

In regions I and III, the vacuum solutions that satisfy the required boundary conditions $\left(\phi_{\mathrm{I}}\left(W_{1}\right)=0\right.$ and $\left.\phi_{\mathrm{III}}\left(W_{2}\right)=0\right)$ are given by the usual solutions to the Laplace equation in the form

$\phi_{\mathrm{I}}(r)=A\left(r^{m}-\frac{W_{1}^{2 m+1}}{r^{m+1}}\right)$

$\phi_{\mathrm{III}}(r)=B\left(r^{m}-\frac{W_{2}^{2 m+1}}{r^{m+1}}\right)$,

where $A$ and $B$ are two constants to be determined by matching the boundary conditions at the interfaces between plasma and vacuum. Because no surface charges accumulate on these interfaces (the equilibrium density profile should vanish at $R_{1}$ and $R_{2}$ ), the electric field is continuous in the whole space. The matching conditions are, therefore, continuity of $\phi$ and of its first derivatives, namely

$\phi_{\mathrm{I}}\left(R_{1}\right)=\phi_{\mathrm{II}}\left(R_{1}\right)$

$\phi_{\mathrm{I}}^{\prime}\left(R_{1}\right)=\phi_{\mathrm{II}}^{\prime}\left(R_{1}\right)$

$\phi_{\mathrm{III}}\left(R_{2}\right)=\phi_{\mathrm{II}}\left(R_{2}\right)$

$\phi_{\mathrm{III}}^{\prime}\left(R_{2}\right)=\phi_{\mathrm{II}}^{\prime}\left(R_{2}\right)$.

We eliminate $A$ and $B$ from these equations to get the boundary conditions for $\phi_{\mathrm{II}}$. We find that in the plasma column, the potential has to satisfy mixed boundary conditions of the Robin type,

$\alpha_{1 / 2} \phi_{\mathrm{II}}\left(R_{1 / 2}\right)+\beta_{1 / 2} \phi_{\mathrm{II}}^{\prime}\left(R_{1 / 2}\right)=0$,

with the coefficients given by

$\alpha_{1 / 2}=\left(m R_{1 / 2}^{m-1}+(m+1) \frac{W_{1 / 2}^{2 m+1}}{R_{1 / 2}^{m+2}}\right)$

$\beta_{1 / 2}=-\left(R_{1 / 2}^{m}-\frac{W_{1 / 2}^{2 m+1}}{R_{1 / 2}^{m+1}}\right)$.
The boundary conditions Eq. (32) are the generalisation of the homogeneous Dirichlet case, Eq. (25), when vacuum gaps are present. If the vacuum regions were removed such that $W_{1}=R_{1}$ and $W_{2}=R_{2}$, the coefficients $\beta_{1 / 2}$ in Eq. (32) would vanish, whereas $\alpha_{1 / 2} \neq 0$. We therefore again find the homogeneous boundary conditions of the previous subsection, Eq. (25).

\section{Numerical procedure}

\subsection{Single domain}

In the single-domain decomposition, the plasma is in contact with both the inner and outer walls. Thus $W_{1}=R_{1}$ and $W_{2}=R_{2}$. There is no need to distinguish between $R$ and $W$. The generalised linear eigenvalue problem Eq. (21) is solved numerically by using a pseudo-spectral Galerkin method taking advantage of the homogeneous boundary conditions imposed on the perturbed electric potential, $\phi\left(R_{1 / 2}\right)=0$. First, the independent variable $r$ in Eq. (21) is transformed into a new independent variable $x$ such that $r \in\left[R_{1}, R_{2}\right]$ is mapped into the interval $x \in[-1,1]$.

This coordinate transformation reads:

$r=\frac{R_{2}-R_{1}}{2} x+\frac{R_{2}+R_{1}}{2}$

Second, the unknown eigenfunction $\phi$ is expanded into a set of basis functions $\left(\psi_{k}\right)_{k \geq 2}$ (Boyd 2001) defined for $n \geq 1$ by

$\psi_{2 n}(x) \equiv T_{2 n}(x)-1$

$\psi_{2 n+1}(x) \equiv T_{2 n+1}(x)-x$.

Therefore the boundary conditions are automatically satisfied for each function of this particular basis, $\left.\psi_{k}( \pm 1)\right|_{k \geq 2}=0$. Here $T_{k}(x)=\cos (k \arccos (x))$ are the Tchebyshev polynomials. Let $N$ be the number of collocation points $\left(x_{i}\right)_{0 \leq i \leq N-1}$ for the Tchebyshev expansion:

$x_{i}=\cos \left(\frac{i \pi}{N-1}\right) \quad i=0,1, \ldots, N-1$.

The unknown function is expanded according to

$\phi(x)=\sum_{k=2}^{N-1} \phi_{k} \psi_{k}(x)$.

The matrix discretisation of Eq. (21) leads to the generalised linear eigenvalue problem

\section{$L \phi=\omega M \phi$,}

where we have introduced the unknown vector $\phi=$ $\left(\phi_{2}, \phi_{3}, \ldots, \phi_{N-1}\right)$, and the square matrices are defined by:

$$
\begin{aligned}
& L_{i-1, k-2} \equiv m \Omega\left(x_{i}\right) \mathcal{L}_{m}\left(\psi_{k}\right)\left(x_{i}\right)+q_{m}\left(x_{i}\right) \psi_{k}\left(x_{i}\right) \\
& M_{i-1, k-2} \equiv \mathcal{L}_{m}\left(\psi_{k}\right)\left(x_{i}\right) \\
& i=1,2, \ldots, N-2 \\
& k=2,3, \ldots, N-1 .
\end{aligned}
$$

The system Eq. (40) is then solved by standard routines to compute the eigenvalues and the eigenvectors. 


\subsection{Multi-domain decomposition}

In the multi-domain decomposition, the Galerkin basis in Eqs. (36)-(37) is not adapted anymore. The new basis functions have to satisfy the following Robin boundary conditions:

$\alpha_{1} \psi_{k}(-1)+\beta_{1} \psi_{k}^{\prime}(-1)=0$

$\alpha_{2} \psi_{k}(+1)+\beta_{2} \psi_{k}^{\prime}(+1)=0$.

We look for basis functions expressed by a three-term expansion in Tchebyshev polynomials, for $k \geq 2$, like

$\psi_{k}(x)=T_{k}(x)+a_{k} T_{k-1}(x)+b_{k} T_{k-2}(x)$.

For $\psi_{k}(x)$ to satisfy the conditions Eqs. (43)-(44), the unknowns $a_{k}$ and $b_{k}$ have to be solutions of the system

$a_{k}\left[\beta_{1}(k-1)^{2}-\alpha_{1}\right]+b_{k}\left[\alpha_{1}-\beta_{1}(k-2)^{2}\right]=-\alpha_{1}+\beta_{1} k^{2}$

$a_{k}\left[\beta_{2}(k-1)^{2}+\alpha_{2}\right]+b_{k}\left[\alpha_{2}+\beta_{2}(k-2)^{2}\right]=-\alpha_{2}-\beta_{2} k^{2}$.

The procedure to discretise the eigenvalue problem is the same as the one described in the previous section. We only need to replace the old basis Eqs. (36)-(37) by the new basis Eq. (45).

\section{Results}

Using the aforementioned numerical algorithm, we compute the eigenfunctions and eigenspectra of the diocotron instability for various equilibrium density profiles and charges on the inner wall for laboratory plasmas, Sect. 5.1 and several rotation profiles for pulsar's electrosphere, Sect. 5.2.

In all computations, the external magnetic field follows a decreasing power law with index $\alpha \geq 0$ such that

$B_{z}=\frac{B_{0}}{r^{\alpha}}$

\subsection{Plasma column}

First, we consider the laboratory plasma confined by some external experimental electromagnetic devices. The magnetic field and the density profile are specified as initial data. The rotation profile is then deduced from the electric drift approximation Eq. (17).

\subsubsection{Constant density profile}

The simplest case corresponds to a uniform magnetic field $B_{0}$, $\alpha=0$ and a constant charge density $\rho_{0}$ extending from the origin $r=0$ to the outer wall, so $W_{1}=R_{1}=0$, no inner conducting wall, $W_{2}=R_{2}$, and also $Q=0$ (single domain). In this particular case, the equilibrium speed is constant:

$\Omega=-\frac{\rho_{0}}{2 \varepsilon_{0} B_{0}}$.

The functions $q_{m}$ are all identically zero and the analytical solution to the eigenvalue problem Eq. (40) is simply

$\omega=m \Omega$.

There is no instability. Our numerical algorithm is able to reproduce the eigenvalues Eq. (50) with great accuracy when taking only a few discretisation points (9 points are already enough). The convergence is very fast due to the evanescent truncation error introduced by the Tchebyshev expansion. The precision easily achieves 10 digits.

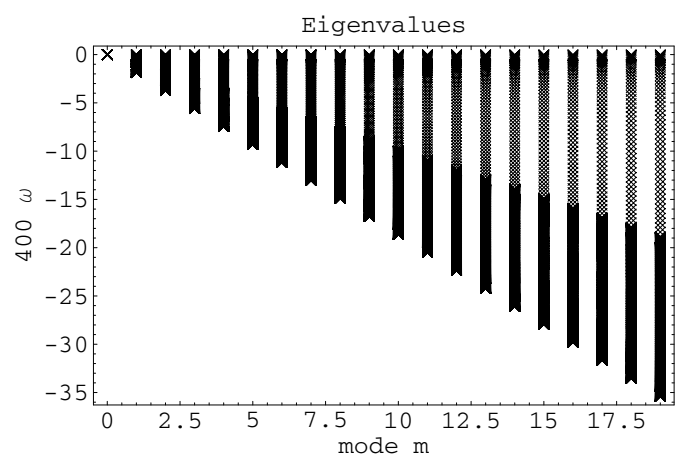

Fig. 1. Eigenvalues for the monotonely decreasing density profile Eq. (51). The boundaries are set to $W_{1}=R_{1}=1$ and $W_{2}=R_{2}=20$. The other parameters are $\alpha=0$ and $Q=0$. The continuous spectrum is shown for the azimuthal modes $m=0,1,2, \ldots, 19$. The eigenfrequencies have been normalised to the rotation frequency $\omega_{r}=\omega_{\mathrm{p}}^{2} / 4 \omega_{\mathrm{c}}=1 / 400$.

\subsubsection{Decreasing density profile}

Next we consider a monotonely decreasing density profile starting from $\rho_{0}$ at $r=W_{1}=R_{1}$ and vanishing at $r=W_{2}=R_{2}$ (single domain) such that

$\rho=\rho_{0} \frac{R_{2}^{2}-r^{2}}{R_{2}^{2}-R_{1}^{2}}$.

The corresponding rotation profile can be found analytically. The electric field induced by the plasma is deduced from Eq. (6), whereas the contribution from the wall is given by Eq. (2). Using the drift approximation Eq. (11), the total radial electric field expressed in Eq. (7) and the power-law index for the magnetic field Eq. (48), the rotation speed of the plasma column in the most general case ( $\alpha$ and $Q$ arbitrary) is equal to

$$
\begin{aligned}
\Omega= & -\frac{\rho_{0} r^{\alpha}}{\varepsilon_{0} B_{0}\left(R_{2}^{2}-R_{1}^{2}\right)}\left[\frac{R_{2}^{2}}{2}\left(1-\frac{R_{1}^{2}}{r^{2}}\right)-\frac{1}{4}\left(r^{2}-\frac{R_{1}^{4}}{r^{2}}\right)\right] \\
& -\frac{Q}{2 \pi \varepsilon_{0} r^{2-\alpha} B_{0}} .
\end{aligned}
$$

When the plasma is in contact with the inner, as well as with the outer, conducting wall and the applied magnetic field is uniform, there is no diocotron instability, Briggs et al. (1970). Indeed, for $\alpha=0$, all the eigenvalues we found are real as expected. For the first azimuthal modes $m \leq 19$, the map of the eigenvalues in the complex plane is shown in Fig. 1 for a uniform magnetic field, $\alpha=0$, zero charge, $Q=0, W_{1}=R_{1}=1$, and $W_{2}=R_{2}=20$. We normalised the frequencies to the rotation frequency $\omega_{\mathrm{r}}=$ $\omega_{\mathrm{p}}^{2} / 4 \omega_{\mathrm{c}}=1 / 400$. Therefore, for each mode, the spectrum is continuous and delimited by two integer values:

$0 \geq \omega_{m} / \omega_{r} \geq-2 m$.

A similar conclusion also applies when the inner wall is removed $\left(W_{1}=R_{1}=0\right)$, see Fig. 2 . In this case the continuous spectra satisfy

$-m \geq \omega_{m} / \omega_{r} \geq-2 m$.

Finally, when switching from a uniform magnetic field to a decreasing power law Eq. (48) with for instance $\alpha=1$, the results are qualitatively unchanged, see Fig. 3. As can be seen by comparing the two maps in Figs. 1 and 3, the radial profile of the magnetic field does not influence the eigenvalues much. There is mainly a difference in scale because the rotation profile for $\alpha=1$ leads to higher rotation rate due to the extra factor $r^{\alpha}$ in Eq. (52). 


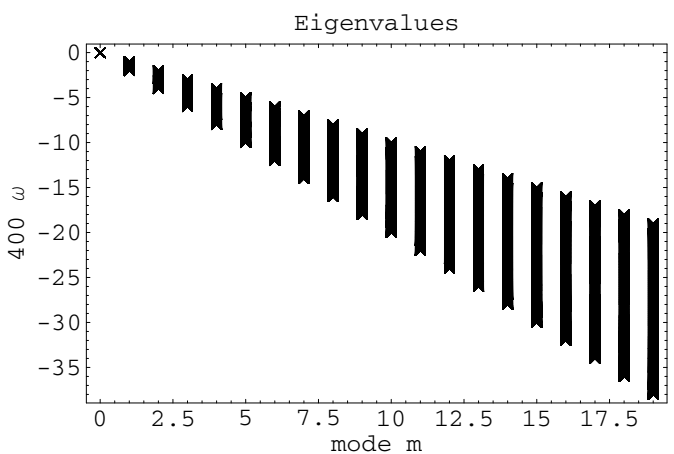

Fig. 2. Same as Fig. 1 but the inner wall has been removed, i.e. $W_{1}=R_{1}=0$.

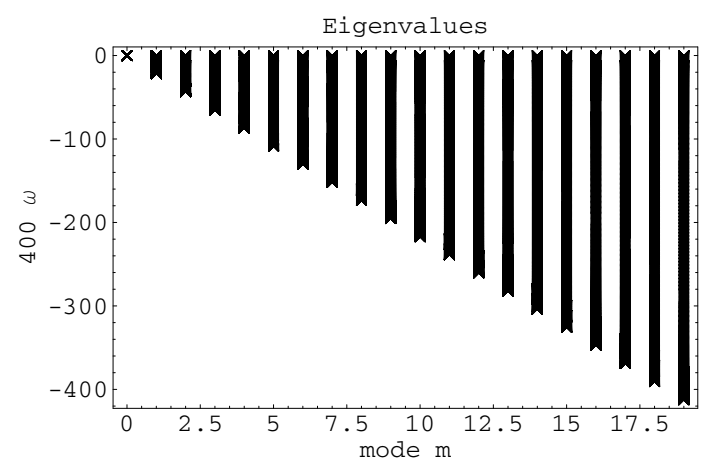

Fig. 3. Same as Fig. 1 but with a decreasing external magnetic field, $\alpha=1$. Note the difference in scale for both cases.

\subsubsection{Gaussian density profile}

Next we consider a situation in which the plasma is not in contact with either the inner or the outer wall so $W_{1}<R_{1}$ and $W_{2}>R_{2}$, (multi-domain decomposition). We choose a Gaussian density profile given by

$\rho=\rho_{0} \mathrm{e}^{-(r-a)^{2} / 2 \sigma^{2}}$

where $\sigma$ is a measure of the profile radial spread while $a$ specifies the location of the centre of the plasma column. The associated rotation rate of the plasma column in the most general case $(\alpha$ and $Q$ arbitrary) is found analytically to be

$$
\begin{aligned}
\Omega= & -\frac{\rho_{0} \sigma}{\varepsilon_{0} B_{0} r^{2-\alpha}}\left[\sigma\left\{\mathrm{e}^{-\left(R_{1}-a\right)^{2} / 2 \sigma^{2}}-\mathrm{e}^{-(r-a)^{2} / 2 \sigma^{2}}\right\}\right. \\
& \left.+a \sqrt{\frac{\pi}{2}}\left\{\operatorname{erf}\left(\frac{r-a}{\sigma \sqrt{2}}\right)-\operatorname{erf}\left(\frac{R_{1}-a}{\sigma \sqrt{2}}\right)\right\}\right]-\frac{Q}{2 \pi \varepsilon_{0} r^{2-\alpha} B_{0}}
\end{aligned}
$$

where $\operatorname{erf}(x)$ is the error function, Abramowitz \& Stegun (1965). In this case, we expect a strong diocotron instability with growth rates of the same order of magnitude as the rotation speed of the perturbation.

Indeed, taking boundaries such that $W_{1}=1, W_{2}=20$, $R_{1}=5$, and $R_{2}=10$ and a uniform magnetic field $\alpha=0$, no charge on the inner wall $Q=0$, the map of the eigenvalues in the complex plane is given by Fig. 4 . Only the low azimuthal unstable modes are excited from $m=1$ to $m=7$, the fastest mode being $m=4$ with $\gamma_{\max } \approx 1.15 \times 10^{-3}$, which has the same order of magnitude as the real part, $\operatorname{Re}(\omega) / m$. Removing the inner wall has little effect on the diocotron spectrum; compare Figs. 4 and 5. Indeed, the instability is initiated by the presence of a hole in the middle of the plasma column. Placing or removing an inner wall cannot suppress or generate the instability in this configuration. It can at most affect the rate at which

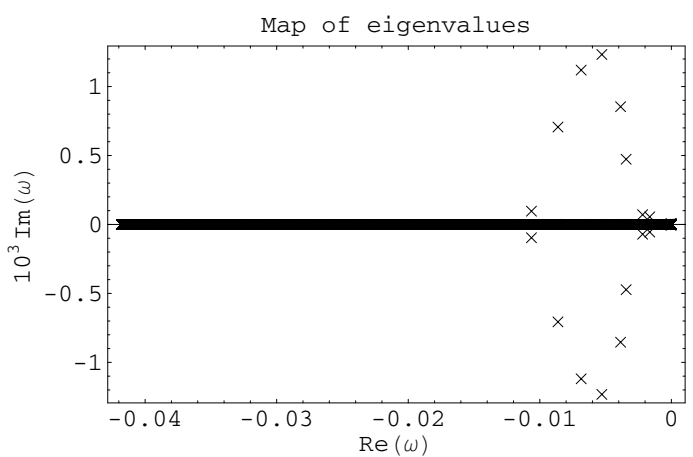

Fig. 4. Eigenvalues for the Gaussian density profile Eq. (55) with $\sigma=1$ and $a=7$. The boundaries are set to $W_{1}=1, W_{2}=20, R_{1}=5, R_{2}=10$, and $\alpha=0$.

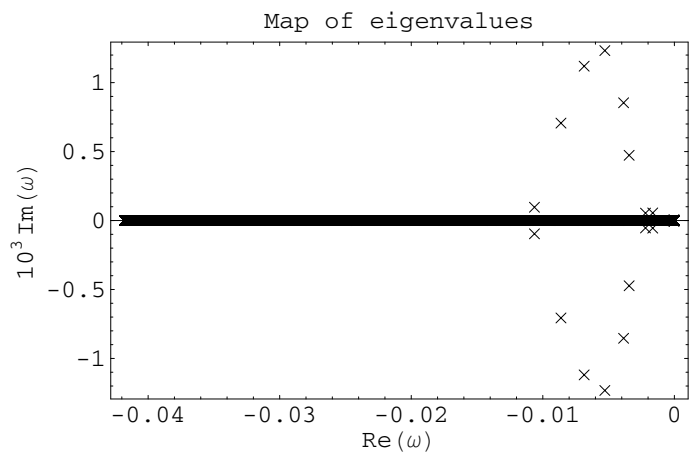

Fig. 5. Same as Fig. 4 but the inner wall has been removed, i.e. $W_{1}=0$.

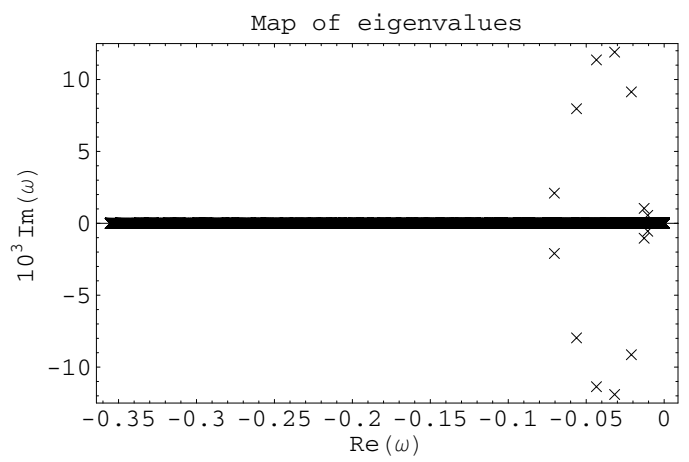

Fig. 6. Same as Fig. 4 but with a decreasing external magnetic field $\alpha=1$.

the instability grows. Also, a decreasing magnetic field $\alpha=1$ does not affect the results in a dramatic way, compare Figs. 4 and 6 . The number of excited modes is directly related to the radial spread of the plasma column. More precisely, the thinner the plasma ring, the larger the number of unstable modes. In other words, decreasing $\sigma$ will generate more unstable modes. In Fig. 7, we compare three Gaussian distributions with different spreads, namely $\sigma=1,0.6,0.4$. The $\sigma=1$ profile has 7 unstable modes, whereas the $\sigma=0.6$ profile has 10 unstable modes and the $\sigma=0.4$ profile 14 . Meanwhile, the highest growth rate is also increasing significantly.

Finally, adding a charge to the inner wall does not affect the map of eigenfrequencies drastically, see Fig. 8. More unstable modes appear, nevertheless the maximum growth rates remain approximately within the same order of magnitude. Now that the algorithm has been checked and employed for different lab plasmas, we study in the next subsection the interesting case of an electrospheric plasma. 


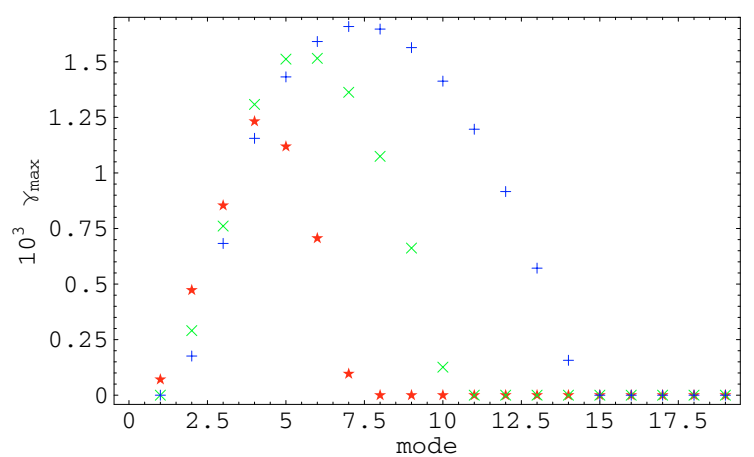

Fig. 7. Comparison of the highest growth rates for different spreads of the annular plasma column, $\sigma=1$ in red "stars", $\sigma=0.6$ in green " $\times$ " and $\sigma=0.4$ in blue "+".

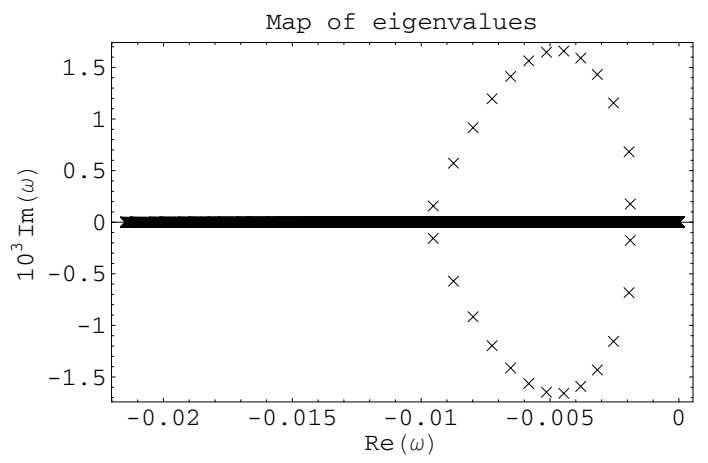

Fig. 8. Same as Fig. 4 but with a charge per unit length $Q=10$.

Table 1. Parameters for the three rotation profiles used to mimics the azimuthal velocity of the plasma in the electrospheric disk.

\begin{tabular}{cccc}
\hline \hline$\Omega$ & $\alpha$ & $\beta$ & $r_{0}$ \\
\hline$\Omega_{1}$ & 3.0 & $5 \times 10^{-5}$ & 6.0 \\
$\Omega_{2}$ & 1.0 & $5 \times 10^{-5}$ & 6.0 \\
$\Omega_{3}$ & 0.3 & $5 \times 10^{-5}$ & 10.0 \\
\hline
\end{tabular}

\subsection{Electrosphere}

The electrospheric non-neutral plasma, as already proved in works by Krause-Polstorff \& Michel (1985a) and Pétri et al. (2002a), is confined by the rotating magnetised neutron star. The most important feature is not the density profile but the rotation speed, although both are related in a unique manner, Eq. (14). Plasma-shearing motion between different magnetic surfaces leads to a kind of Kelvin-Helmholtz instability, at least in the linear regime of the instability (the eigenvalue problems look very similar). (We always keep a single-domain decomposition such that $W_{1}=R_{1}$ and $W_{2}=R_{2}$ ). Therefore, for electrospheric plasmas, we choose a rotation profile in the plasma column that mimics the rotation curve obtained in the $3 \mathrm{D}$ electrosphere. To study the influence of the profile, we took three different analytical expressions for the radial dependence of $\Omega$ by mainly varying the gradient in differential shear as follows

$\Omega(r)=2+\tanh \left[\alpha\left(r-r_{0}\right)\right] \mathrm{e}^{-\beta r^{4}}$.

The values used are listed in Table (1).

The angular velocity starts from corotation with the star $\Omega=\Omega_{*}=1$ (normalised to unity for convenience) followed by a sharp increase around $r=6$ for $\Omega_{1,2}$ and a less pronounced gradient around $r=10$ for $\Omega_{3}$. Finally the rotation rate asymptotes at twice the neutron star rotation speed, Fig. 9.

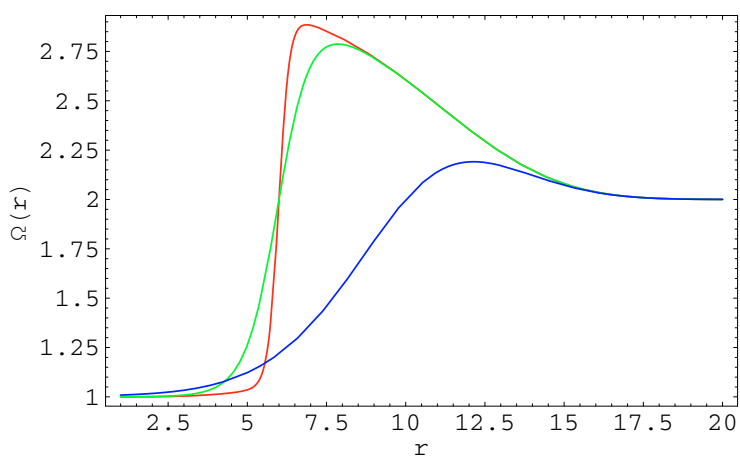

Fig. 9. Three choices of differential rotation curves in the plasma column for the cylindrical pulsar electrosphere, $\Omega_{1}$ in red, $\Omega_{2}$ in green, and $\Omega_{3}$ in blue.

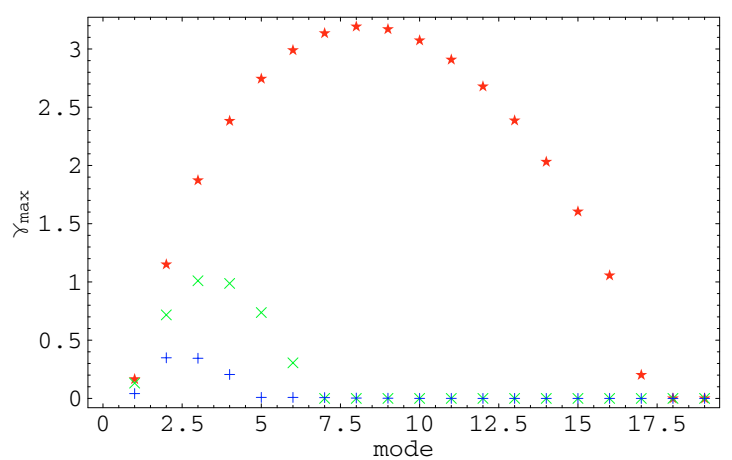

Fig. 10. Fastest growth rate $\gamma_{\max }$ for each azimuthal mode $m$ and the three rotation profile shown in Fig. 9 in a uniform external magnetic field, $\alpha=0, \Omega_{1}$ in red "stars", $\Omega_{2}$ in green "×" and $\Omega_{3}$ in blue "+".

\subsubsection{Uniform magnetic field}

First consider a uniform magnetic field, $\alpha=0$. The maximum growth rates for the three rotation curves for each mode $m$ are shown in Fig. 10.

The profile with the steepest gradient possesses the largest number of excited unstable modes because it corresponds to the case where the smallest scales appear, i.e. $m$ large, red stars in Fig. 10. The highest growth rate, for $m=8$, its value is $\gamma_{\max }=3.2$, is even higher than the maximum rotation speed of the plasma column about $\Omega_{\max }=2.9$. The diocotron instability operates on a very short timescale, comparable to the period of the pulsar.

The second steepest profile possesses less unstable modes as we would expect due to the fact that only larger-scale structures can emerge with this slope of the differential rotation, green " $x$ " in Fig. 10. The third smooth profile has only four unstable modes, blue "+" in Fig. 10. We give an example of eigenfunctions for the perturbed electric potential in Fig. 11. It corresponds to the fastest unstable mode for the profile $\Omega_{2}$. The boundary conditions impose $\phi\left(R_{1 / 2}\right)=0$, as seen on the plot. The corotation radius $r_{\mathrm{c}}$ defined by

$\Omega\left(r_{\mathrm{c}}\right)=\frac{\operatorname{Re}(\omega)}{m}$

is also shown on this plot, depicted by a vertical bar. 


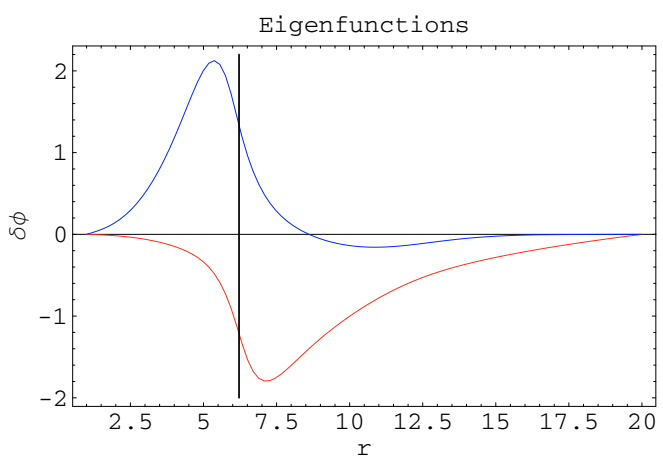

Fig. 11. Real (red curve) and imaginary (blue curve) parts of the fastest growing eigenfunction for $\Omega_{2}$ in a uniform magnetic field. The vertical bar shows the location of the corotation radius.

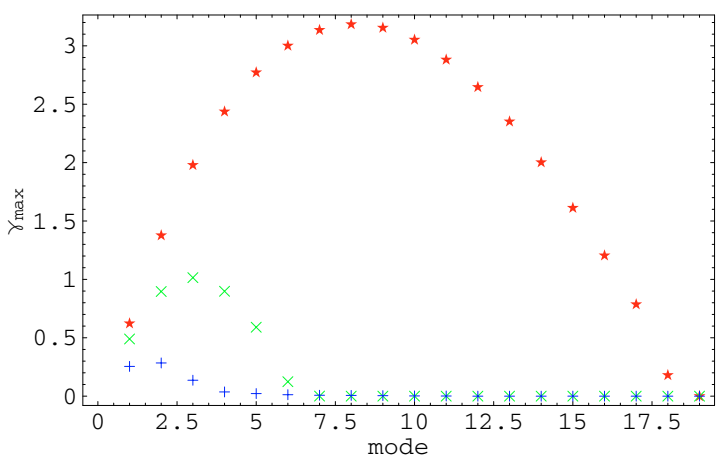

Fig. 12. Fastest growth rate $\gamma_{\max }$ for each azimuthal mode $m$ and the three rotation profile shown in Fig. 9 in a dipolar external magnetic field, $\alpha=3, \Omega_{1}$ in red "stars", $\Omega_{2}$ in green "X", and $\Omega_{3}$ in blue "+".

\subsubsection{Dipolar magnetic field}

Next consider a dipolar magnetic field, $\alpha=3$. The maximum growth rates for the three rotation curves for each mode $m$ are shown in Fig. 12.

We can draw the same conclusions as in the previous case. The largest number of excited unstable modes is observed for the steepest profile $\Omega_{3}$. Comparing Figs. 12 with 10, the value of the maximum growth rate for each mode is not strongly affected by the geometry of the magnetic field. Here also, we give an example of eigenfunctions for the perturbed electric potential in Fig. 13. It again corresponds to the fastest unstable mode for the profile $\Omega_{2}$. The boundary conditions impose $\phi\left(R_{1 / 2}\right)=0$ as seen on the plot. Comparing Figs. 13 with 11, close to the inner boundary, the real part of the eigenfunctions look very similar. Nevertheless when approaching the outer boundary, the difference becomes appreciable because of the strong departure from a uniform magnetic field.

\section{Conclusion}

We have developed a pseudo-spectral Galerkin code to compute the eigenspectra and eigenfunctions of the diocotron instability for arbitrary magnetic-field configurations and density profiles. The code is very efficient in computing the fastest growing modes. When increasing the number of points, convergence is reached quickly; and in most cases, less than 200 points are needed. Application to the pulsar shows that the diocotron regime gives rise to instabilities with a growth rate comparable to the rotation period of the neutron star. This confirms the results of our previous work (Pétri et al. 2002b) and shows that

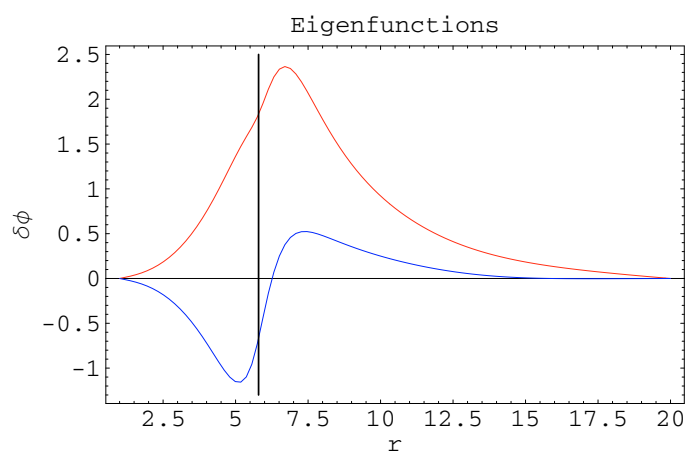

Fig. 13. Real (red curve) and imaginary (blue curve) part of the fastest growing eigenfunction for $\Omega_{2}$ in a dipolar magnetic field. The vertical bar shows the location of the corotation radius.

the precise geometry, whether a flat disk or a column of plasma, does not drastically affect the diocotron instability. The required ingredients are only non-neutral plasmas and shear velocities.

In a forthcoming paper, we plan to investigate the full nonlinear development of the diocotron instability by means of 2D electrostatic PIC simulations. We will also add an external source of charge feeding the system and demonstrate that particle transport across the magnetic field line is possible. Note that this has already been observed in some experiments by Pasquini \& Fajans (2002).

Extension to plasma moving with relativistic speeds is also envisageable, in particular the generalisation to the electromagnetic non-neutral instability regime, the so-called magnetron instability. Last but not least, the influence of finite temperature in the plasma on the diocotron instability would require a kinetic treatment of the stability via the Vlasov-Maxwell equation.

Acknowledgements. I am grateful to Jean Heyvaerts for his helpful comments and suggestions. This work was supported by a grant from the G.I.F., the German-Israeli Foundation for Scientific Research and Development.

\section{References}

Abramowitz, M. \& Stegun, I. A. 1965, Handbook of mathematical functions (New York: Dover)

Aly, J. J. 2005, A\&A, 434, 405

Bhattacharyya, S. N. 2000, Phys. Plasmas, 7, 4805

Boyd, J. P. 2001, Chebyshev and Fourier Spectral Methods (Springer-Verlag) Briggs, R. J., Daugherty, J. D., \& Levy, R. H. 1970, Phys. Fluids, 13(2), 421

Davidson, R. C. 1990, Physics of non neutral plasmas (Addison-Wesley Publishing Company)

Davidson, R. C., \& Tsang, K. 1984, Phys. Rev. A, 30, 488

Goswami, P., Bhattacharyya, S. N., Sen, A., \& Maheshwari, K. P. 1999, Phys. Plasmas, 6, 3442

Krause-Polstorff, J., \& Michel, F. C. 1985a, MNRAS, 213, 43P

Krause-Polstorff, J., \& Michel, F. C. 1985b, A\&A, 144, 72

Levy, R. H. 1965, Phys. Fluids, 8, 1288

Michel, F. C. 2005, Rev. Mex. Astron. Astrofis. Conf. Ser., 27

Neukirch, T. 1993, A\&A, 274, 319

O’Neil, T. M. 1980, Phys. Fluids, 23, 2216

O’Neil, T. M., \& Smith, R. A. 1992, Phys. Fluids B, 4, 2720

Pasquini, T., \& Fajans, J. 2002, AIP Conf. Proc. 606: Non-Neutral Plasma Physics IV, 453

Pétri, J., Heyvaerts, J., \& Bonazzola, S. 2002a, A\&A, 384, 414

Pétri, J., Heyvaerts, J., \& Bonazzola, S. 2002b, A\&A, 387, 520

Pétri, J., Heyvaerts, J., \& Bonazzola, S. 2003, A\&A, 411, 203

Rylov, I. A. 1989, Ap\&SS, 158, 297

Shibata, S. 1989, Ap\&SS, 161, 187

Smith, I. A., Michel, F. C., \& Thacker, P. D. 2001, MNRAS, 322, 209

Spitkovsky, A., \& Arons, J. 2002, Neutron Stars in Supernova Remnants, ASP Conf. Ser., 271, 81

Thielheim, K. O., \& Wolfsteller, H. 1994, ApJ, 431, 718

Zachariades, H. A. 1993, A\&A, 268, 705 